\title{
Biomass Production and Carbon Sequestration by Pongamia pinnata (Linn) Pierre in Tropical Environment
}

\author{
Priyanka Bohre ${ }^{1}$, O. P. Chaubey ${ }^{1}$ and P. K. Singhal ${ }^{2}$ \\ ${ }^{1}$ State Forest Research Institute, Jabalpur - 482008, M.P. India \\ ${ }^{2}$ Rani Durgawati University, Jabalpur- 482001, M.P. India \\ pribohre@gmail.com,chaubey.dr@gmail.com,pksinghalrdvv@gmail.com
}

\begin{abstract}
The significance of role of biomass of tree species in carbon sequestration has long been recognized. In the present paper, attempts have been made to work out biomass accumulation and carbon sequestration by Pongamia pinnata (Linn) Pierre raised on coal mine overburden of Northern Coal Field Limited, Singrauli (India), adopting non harvest technique. The age of plantation varied from 2 to 18 years. The correlation between basal area vs volume, dbh vs volume and basal area vs total biomass was found to be significant. In terms of vertical and horizontal growth, Pongamia pinnata proved as an efficient species. The net biomass production and carbon sequestration of Pongamia pinnata showed increasing trend with the age of plantations.
\end{abstract}

Keywords: Carbon sequestration, biomass accumulation, non harvest technique, allometric equation, plantation forests

\section{Introduction}

In the current scenario of climate change (Manua Loa observatory 2013) and Reducing Emissions from Deforestation and Forest Degradation (REDD) and REDD plus (ISFR 2011; Ravindranath et al., 2012), eco-restoration on degraded ecosystems is the need of the hour. National Afforestation Programme (NAP) and National Mission for Green India (GIM) are being implemented in the country. It is estimated that addition or improvement of forest and tree cover by implementing the various forestry related programmes will add 2 million tonnes of carbon incrementally every year, and post 2020, the forest and tree cover will be adding at least 20 million tonnes of carbon every year (Sud et al., 2012; GIM Report 2012). The National Afforestation Programme (NAP) aims to restore degraded forests.

Because of importance of carbon pools in tropical forests and in the plantation systems, many organizations around the world carried out studies related to forest biomass and carbon storage in recent decades. Many workers studied biomass production of tropical forests and different species by actual harvest at a predetermined age and allometric equations relating biomass with one or more tree dimensions (Kira et al. 1964; Ogawa et al., 1965; Hozumi et al., 1969; Odum and Pigeon 1970; Jordan 1971; Whitemore 1975; Edwards and Grubbs 1977; Enright 1979; Tanner 1980; Negi et al., 1984; Prasad and Mishra 1984; Prasad et al., 1984; Rai 1984; Sharma and Srivastava 1984; Chaturvedi 1984). The results might be quite 
different, if the age of assessments had changed. Generation of allometric equations that involve felling of trees is impracticable in the current scenario of climate change. Thus, it is required to adopt non harvest techniques and acceptable level of accuracy. The present paper deals with the suitability of Pongamia pinnata for biomass accumulation and carbon sequestration in the degraded ecosystem of coal mine.

\section{Materials and Methods}

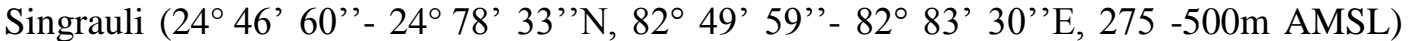
was granted District Status on $24^{\text {th }}$ May 2008, with its headquarter at Waidhan. Vegetation during pre-mining period was very dense and covered with Northern tropical dry sal forests $(5$ $\mathrm{B} / \mathrm{C}$ ) and Northern tropical dry mixed deciduous forests (5 B/C 2). Due to mining, the large forest areas were clear felled and laid barren. The present study covered artificial plantations raised in eight open cast project (OCP) sites viz; Jayant, Dudhichua, Amlohri, Jhingurdah, Nigahi, Bina, Kakari and Khadia of Northern Coal field Limited (NCL) Singrauli, India (Figure 1).

For the estimation of biomass, non harvest technique was adopted. The growth data was collected for total height and girth at breast height (gbh) over bark. Besides, the length of tree after gbh had been divided into different segments of one meter each up to the tip of the tree. The girth at over bark of each segment was measured at the center of the segments throughout the height of the tree for minimizing the tapering effect. The girth was measured without felling trees with the help of climbers. Girth was converted to diameter by dividing $\pi$, i.e., 3.14. Volume was calculated for each imaginary segment using cylindrical cross sectional areas, multiplied by height of each segment $\left(\pi r^{2} h\right)$. Total volume of the bole was worked out by adding the volumes of different imaginary segments starting from gbh to top of a height and the volume of the base segment (i.e., below gbh). The dbh, total height and total volumes were fed in SPSS software using computer. On the basis of the maximum coefficient of determination $\left(\mathrm{R}^{2}\right)$ and the minimum standard error, the best fit model was computed for the species. Multiple regression equations were tried to establish the correlation between the dbh and height, between dbh and volume, and the biomass and dbh.

The stem wood biomass was worked out by multiplying volume with wood density (Reyes et al., 1992) of Pongamia pinnata. The stem wood biomass was then "expanded" to total above ground biomass of tree including leaves, twigs, branches, bole and bark using biomass expansion factor (BEF).

Total above ground biomass $=$ Stem wood volume $\mathrm{X}$ Wood density $\mathrm{X}$ BEF

The mean BEF value of 1.5 was used for this study as prescribed by Brown and Luge (1992). The below ground biomass was calculated by using simple default value of $25 \%$ (for hardwood species) of the total above ground biomass as recommended by IPCC (2006). Wood density information was presented in units of oven dry weight in $\mathrm{gm}^{-3}$ (i.e., tonne $\mathrm{m}^{-3}$ ) of green volume. Multiple regression equations were tried to establish the correlation between the biomass and dbh and / or bole biomass. The amount of carbon in a standing tree was calculated by dividing its biomass by 2 as per the guidelines of IPCC (2006), and was expressed in tonne tree ${ }^{-1}$ and tonne $\mathrm{ha}^{-1}$. Carbon content was then multiplied by 44/12 to estimate $\mathrm{CO}_{2}$.

Nursery raised seedlings were used for this purpose. Fifteen randomly selected seedlings of each species were harvested for measuring their height and dry weight (dried at $104^{\circ} \mathrm{C}$ till the constant weight obtained). The average height and dbh of each species according to age were taken to estimate volume. The best fit equation was then applied to determine the 
accumulation of total biomass (above- and below- ground) and carbon content. The initial value of biomass and carbon in a seedling of a species was then subtracted from its corresponding estimates to obtain realistic amount of biomass accumulation and carbon sequestration by a tree. Per tree accumulation of biomass was multiplied by the actual number of seedlings usually planted per hectare (i.e., 3333) to express the values in tonne ha ${ }^{-1}$. The biomass and carbon tables were prepared after making volume growth tables. The best fit regression equation was determined using SPSS software to prepare volume growth tables. The general volume equations (GVEs), i.e. regression function in volume diameter and height, were selected for each species. The nine regression equations, as used by Forest Survey of India (FSI 1996), were attempted to determine the best equation for estimating volume overbark (VOB) for this species. After getting values of constants for best fit equation, the actual volumes as well as those predicted by the equation were tabulated and computed. The biomass tables for above - and below- ground biomass were prepared separately for different diameter and height classes for Pongamia pinnata. The table for the total biomass of a tree was obtained by adding its values of above and below ground biomass. The projected biomass tables for above- and below- ground and total biomass were also prepared using data of volumes estimated for tree of different $\mathrm{dbh}(2 \mathrm{~cm}$ interval $)$ and height ( $1 \mathrm{~m}$ interval) class. The biomass was expressed in tonne tree ${ }^{-1}$.

\section{Results}

One hundred three trees (each having $\geq 5 \mathrm{~cm} \mathrm{dbh}$ ) were randomly selected from total trees to quantify their biomass and carbon. The diameter and girth at breast height varied widely from 6.36 to $34.36 \mathrm{~cm}$ and 20 to $108 \mathrm{~cm}$ mainly due to variation in the age of tree in different plantations. Total height ranged from 2 to $17.32 \mathrm{~m}$. The height varied significantly within a gbh or dbh class, denoting that vertical growth of trees varied among different sites due to variation in growth factors. For example, the height of trees with dbh of $7.00 \mathrm{~cm}$ varied from 3 to $9 \mathrm{~m}$. Such variations were observed in all age classes. The volume of trees varied positively and linearly in response to variation in its basal area $\left(r=0.953, r^{2}=0.909\right)$. The variation in basal area could explain nearly $91 \%$ of the variation in volume. Therefore, basal area could be a good predictor of volume in trees. The total biomass of trees varied positively and linearly with variation in its basal area $\left(r=953, r^{2}=0.909\right)$. Basal area explained a higher proportion (i.e. 91\%) of variation recorded in total biomass. Though, diameter at breast height was used to estimate basal area, it could explain relatively lower amount of variation in volume ( $\left.\mathrm{r}=0.940, \mathrm{r}^{2}=0.884\right)$. dbh could hardly explain $88 \%$ of variation recoded in volume of trees. The minimum and maximum volume values of trees were computed between 0.00781 $\mathrm{m}^{3}$ and $0.40646 \mathrm{~m}^{3}$, minimum and maximum total biomass values of trees ranged between 0.008347 tonne tree ${ }^{-1}$ and 0.434401 tonne tree ${ }^{-1}$ and the value of carbon sequestered varied from 0.004174 (minimum) to 0.2172000 tonne $\operatorname{tree}^{-1}$ (maximum), respectively. The linear correlation between basal area and volume, dbh and volume, and basal area and total biomass among 103 trees taken for actual measurement was found to be significant with the values of $\mathrm{R}^{2}$ being $0.909,0.884$ and 0.909 , respectively. The values of $\mathrm{R}^{2}$ are closer to 1 , which indicates that the better the line fits the data (Figure 2).

Trees measured for estimation of biomass accumulation and carbon sequestration were taken from 2, 4, 5, 6, 8, 9, 10, 13, 14, 15 and 18 years old plantations. The seedlings used for plantations had the average height of $0.55 \mathrm{~m}$. The average dbh in different year's plantations 
showed increasing trend with the advancement of age. In different aged plantations of $2,4,5$, $6,8,9,10,13,14,15$ and 18 years, the average dbh were $2.5 \mathrm{~cm}, 3.6 \mathrm{~cm}, 5.5 \mathrm{~cm}, 6.7 \mathrm{~cm}, 8.8$ $\mathrm{cm}, 10.2 \mathrm{~cm}, 11.5 \mathrm{~cm}, 15.0 \mathrm{~cm}, 16.9 \mathrm{~cm}, 19.7 \mathrm{~cm}$ and $27.8 \mathrm{~cm}$ and average height were $2.50 \mathrm{~m}, 3.10 \mathrm{~m}, 4.65 \mathrm{~m}, 4.80 \mathrm{~m}, 5.10 \mathrm{~m}, 6.10 \mathrm{~m}, 6.50 \mathrm{~m}, 8.10 \mathrm{~m}, 8.35 \mathrm{~m}, 9.20 \mathrm{~m}$ and $12.15 \mathrm{~m}$, respectively. The values of above ground and below ground biomass, and total biomass and carbon content were given for different years old plantations determined by using best fit equation used for individual trees of different $\mathrm{dbh}$ and height (Table 1). On critical examination of the data, the biomass accumulation from the seedling stage to sapling and tree stage in plantations of $2,4,5,6,8,9,10,13,14,15$ and 18 years was found to be 3.90, 4.43, $26.88,43.58,73.88,106.47,135.81,247.32,310.61,434.43$ and 936.42 tonne ha $^{-1}$, respectively, showing the increasing trend of biomass accumulation (Table 2).

Multiple regression equations were tried to find out best fit equation. On the basis of maximum correlation coefficient $\left(\mathrm{R}^{2}\right)$ and minimum standard error, the best model was computed to be VOB $=-0.007+0.002 \mathrm{D}+2.638 \times 10^{-5} \mathrm{D}^{2} \mathrm{H}-3.863 \times 10^{-10}\left(\mathrm{D}^{2} \mathrm{H}\right)^{2}$, Where, $\mathrm{VOB}=$ Volume over bark in $\mathrm{CMT}, \mathrm{D}=$ Diameter at breast height in $\mathrm{cm}, \mathrm{H}=$ Total tree height in $\mathrm{m}$. The general volume table was prepared by using the best fit regression equations on the actual growth data. Variables all entering or removing variables, reveals the independent variables namely $\mathrm{D}, \mathrm{D}^{2} \mathrm{H}$ and $\left(\mathrm{D}^{2} \mathrm{H}\right)^{2}$ are part of the equation and $\mathrm{V}$ is the dependent variable. The model summary of the output of the volume of Pongamia pinnata trees is a function of the multiple correlation coefficient $\left(R=0.970, R^{2}=0.940, S E=0.0235515041\right)$ had its $D, D^{2} H$ and $\left(D^{2} H\right)^{2}$. The ANOVA confirmed that regression of $\mathrm{V}$ on $\mathrm{D}, \mathrm{D}^{2} \mathrm{H}$ and $\left(\mathrm{D}^{2} \mathrm{H}\right)^{2}$ was highly significant $(\mathrm{Fp}<0.001)$. This denotes that one variability in volume of Pongamia pinnata trees is directly regulated by independent variables such as $D, D^{2} H$ and $\left(D^{2} H\right)^{2}$. The coefficients of the output give us the values that we need in order to write the regression equation. The correlation between actual and computed volume for 103 trees, was found to be highly significant at 0.01 levels ( $97 \%$ confidence level).

Since the accuracy of the volume table has been tested statistically, thus it can be safely used to predict the volume of single trees of different dimensions, in efficient and scientific forest management. The general volume table was used for preparing the above ground biomass using the formula: wood density $\left(\mathrm{g} \mathrm{cm}^{-3}=\right.$ tonne $\left.\mathrm{m}^{-3}\right) \mathrm{x}$ volume of tree $\left(\mathrm{m}^{3}\right)$. Biomass table showed the increasing trend between biomass and growth (height and dbh) parameters. The total minimum and maximum biomass was found to be 0.0021 and 0.5571 tonne tree $^{-1}$, respectively. The minimum and maximum values of carbon content were 0.0010 tonne and 0.2786 tonne tree ${ }^{-1}$, respectively. The minimum and maximum values of $\mathrm{CO}_{2}$ absorbed from the atmosphere were 0.0038 and 1.0214 tonne tree $^{-1}$, respectively (Table 3 ). 
Table 1. Biomass and carbon content in Pongamia pinnata according to age of the plantations (values are mean \pm standard deviation)

\begin{tabular}{|c|c|c|c|c|c|c|c|c|c|}
\hline $\begin{array}{l}\text { S. } \\
\text { N. }\end{array}$ & $\begin{array}{c}\text { Plantation } \\
\text { year }\end{array}$ & $\begin{array}{c}\text { Age } \\
\text { (years) }\end{array}$ & $\begin{array}{l}\text { Av. dbh } \\
\text { (cm) }\end{array}$ & $\begin{array}{c}\text { Av. } \\
\text { Height } \\
\text { (m) }\end{array}$ & $\begin{array}{c}\text { Above } \\
\text { ground } \\
\text { biomass } \\
(\text { Tonne } \\
\text { ha-1) }^{-1}\end{array}$ & $\begin{array}{c}\text { Below } \\
\text { ground } \\
\text { biomass } \\
\text { (Tonne } \\
\text { ha-1) }^{-1}\end{array}$ & $\begin{array}{c}\text { Total } \\
\text { biomass } \\
\text { (Tonne } \\
\text { ha- }^{-1} \text { ) }\end{array}$ & $\begin{array}{c}\text { Carbon } \\
\text { content } \\
\text { (Tonne } \\
\text { ha }^{-1} \text { ) }\end{array}$ & $\begin{array}{c}\mathrm{CO}_{2} \\
\text { (Tonne } \\
\text { ha }^{-1} \text { ) }\end{array}$ \\
\hline \multicolumn{2}{|c|}{$\begin{array}{l}\text { Seedling used for } \\
\text { planting }\end{array}$} & $\begin{array}{c}1 / 2 \\
(6 \text { month })\end{array}$ & - & 0.55 & 0.009 & 0.003 & 0.012 & 0.006 & 0.022 \\
\hline 1 & 2007-08 & 2 & $\begin{aligned} & 2.5 \\
& \pm 0.40 \\
&\end{aligned}$ & $\begin{array}{l}2.50 \\
\pm 0.57 \\
\end{array}$ & $\begin{array}{c}3.12 \\
\pm 0.81 \\
\end{array}$ & $\begin{array}{l}0.78 \\
\pm 0.20 \\
\end{array}$ & $\begin{array}{r}3.92 \\
\pm 1.01 \\
\end{array}$ & $\begin{array}{r}1.95 \\
\pm 0.50 \\
\end{array}$ & $\begin{array}{c}7.17 \\
\pm 1.86 \\
\end{array}$ \\
\hline 2 & $2002-03$ & 4 & $\begin{aligned} & 3.6 \\
& \pm 0.62 \\
&\end{aligned}$ & $\begin{array}{c}3.10 \\
\pm 0.42\end{array}$ & $\begin{array}{c}3.56 \\
\pm 1.06 \\
\end{array}$ & $\begin{array}{r}0.89 \\
\pm 0.26 \\
\end{array}$ & $\begin{array}{r}4.44 \\
\pm 1.32 \\
\end{array}$ & $\begin{array}{r}2.22 \\
\pm 0.66 \\
\end{array}$ & $\begin{array}{c}8.15 \\
\pm 2.43 \\
\end{array}$ \\
\hline 3 & 2004-05 & 5 & $\begin{array}{c}5.5 \\
\pm 0.66 \\
\end{array}$ & $\begin{array}{c}4.65 \\
\pm 0.92 \\
\end{array}$ & $\begin{array}{l}21.51 \\
\pm 7.24 \\
\end{array}$ & $\begin{array}{r}5.39 \\
\pm 1.81 \\
\end{array}$ & $\begin{array}{l}26.90 \\
\pm 9.06 \\
\end{array}$ & $\begin{array}{l}13.44 \\
\pm 4.53 \\
\end{array}$ & $\begin{array}{c}49.31 \\
\pm 16.61 \\
\end{array}$ \\
\hline 4 & 2003-04 & 6 & $\begin{array}{c}6.7 \\
\pm 0.72\end{array}$ & $\begin{array}{c}4.80 \\
\pm 0.87\end{array}$ & $\begin{array}{l}34.86 \\
\pm 5.62\end{array}$ & $\begin{array}{c}8.72 \\
\pm 1.40\end{array}$ & $\begin{array}{l}43.59 \\
\pm 7.02\end{array}$ & $\begin{array}{l}21.80 \\
\pm 3.51 \\
\end{array}$ & $\begin{array}{c}79.91 \\
\pm 12.88 \\
\end{array}$ \\
\hline 5 & 2001-02 & 8 & $\begin{array}{r}8.8 \\
\pm 1.08\end{array}$ & $\begin{array}{c}5.10 \\
\pm 0.98\end{array}$ & $\begin{array}{c}59.12 \\
\pm 11.03\end{array}$ & $\begin{array}{l}14.78 \\
\pm 2.75\end{array}$ & $\begin{array}{r}73.89 \\
\pm 13.79\end{array}$ & $\begin{array}{l}36.95 \\
\pm 6.89\end{array}$ & $\begin{array}{l}135.47 \\
\pm 25.29\end{array}$ \\
\hline 6 & 2000-01 & 9 & $\begin{array}{c}10.2 \\
\pm 1.64\end{array}$ & $\begin{array}{l}6.10 \\
\pm 1.10\end{array}$ & $\begin{array}{c}85.19 \\
\pm 16.37\end{array}$ & $\begin{array}{l}21.30 \\
\pm 4.09\end{array}$ & $\begin{array}{l}106.49 \\
\pm 20.46\end{array}$ & $\begin{array}{c}53.25 \\
\pm 10.23\end{array}$ & $\begin{array}{l}195.23 \\
\pm 37.51\end{array}$ \\
\hline 7 & $1999-00$ & 10 & $\begin{array}{r}11.5 \\
\pm 1.25\end{array}$ & $\begin{array}{c}6.50 \\
\pm 1.26\end{array}$ & $\begin{array}{l}108.66 \\
\pm 20.68\end{array}$ & $\begin{array}{l}27.17 \\
\pm 5.17\end{array}$ & $\begin{array}{l}135.83 \\
\pm 25.85\end{array}$ & $\begin{array}{c}67.91 \\
\pm 12.92\end{array}$ & $\begin{array}{l}249.02 \\
\pm 47.39\end{array}$ \\
\hline 8 & $1996-97$ & 13 & $\begin{array}{c}15.0 \\
\pm 1.43\end{array}$ & $\begin{array}{c}8.10 \\
\pm 1.36\end{array}$ & $\begin{array}{l}197.87 \\
\pm 33.12\end{array}$ & $\begin{array}{l}49.47 \\
\pm 8.28\end{array}$ & $\begin{array}{l}247.34 \\
\pm 41.41\end{array}$ & $\begin{array}{l}123.68 \\
\pm 20.70\end{array}$ & $\begin{array}{l}453.45 \\
\pm 75.92\end{array}$ \\
\hline 9 & $1995-96$ & 14 & $\begin{array}{c}16.9 \\
\pm 1.73\end{array}$ & $\begin{array}{c}8.35 \\
\pm 1.90\end{array}$ & $\begin{array}{l}248.49 \\
\pm 25.02\end{array}$ & $\begin{array}{l}62.13 \\
\pm 6.25\end{array}$ & $\begin{array}{l}310.62 \\
\pm 31.28\end{array}$ & $\begin{array}{r}155.31 \\
\pm 15.64\end{array}$ & $\begin{array}{l}569.46 \\
\pm 57.35\end{array}$ \\
\hline 10 & 1994-95 & 15 & $\begin{array}{c}19.7 \\
\pm 1.81\end{array}$ & $\begin{array}{c}9.20 \\
\pm 2.20\end{array}$ & $\begin{array}{l}347.57 \\
\pm 45.92\end{array}$ & $\begin{array}{c}86.90 \\
\pm 11.48\end{array}$ & $\begin{array}{l}434.45 \\
\pm 57.40\end{array}$ & $\begin{array}{l}217.23 \\
\pm 28.70\end{array}$ & $\begin{array}{r}796.50 \\
\pm 105.25\end{array}$ \\
\hline 11 & $1991-92$ & 18 & $\begin{array}{r}27.8 \\
\pm 3.52 \\
\end{array}$ & $\begin{array}{l}12.15 \\
\pm 2.59\end{array}$ & $\begin{array}{l}749.15 \\
\pm 96.97 \\
\end{array}$ & $\begin{array}{l}187.29 \\
\pm 24.24 \\
\end{array}$ & $\begin{array}{c}936.44 \\
\pm 121.21 \\
\end{array}$ & $\begin{array}{l}468.21 \\
\pm 60.60 \\
\end{array}$ & $\begin{array}{l}1716.80 \\
\pm 222.23 \\
\end{array}$ \\
\hline
\end{tabular}

Table 2. Net accumulation of biomass and carbon during growth of Pongamia pinnata in plantation forests

\begin{tabular}{|c|c|c|c|c|c|c|c|}
\hline \multirow[t]{2}{*}{$\begin{array}{l}\text { S. } \\
\text { N. }\end{array}$} & \multirow[t]{2}{*}{$\begin{array}{l}\text { Plantation } \\
\text { year }\end{array}$} & \multirow[t]{2}{*}{$\begin{array}{c}\text { Age } \\
\text { (years) }\end{array}$} & \multicolumn{5}{|c|}{$\begin{array}{l}\text { Biomass and carbon content accumulation after planting } \\
\text { (Biomass accumulation - Biomass of seedling) }\end{array}$} \\
\hline & & & $\begin{array}{c}\text { Above } \\
\text { ground } \\
\text { biomass } \\
\left(\text { Tonne ha-1) }^{-1}\right)\end{array}$ & $\begin{array}{c}\text { Below } \\
\text { ground } \\
\text { biomass } \\
\left(\text { Tonne ha-1) }^{-1}\right)\end{array}$ & $\begin{array}{c}\text { Total } \\
\text { biomass } \\
\left(\text { Tonne ha- }^{-1}\right)\end{array}$ & $\begin{array}{c}\text { Carbon } \\
\text { (Tonne ha-1) }\end{array}$ & $\begin{array}{c}\mathrm{CO}_{2} \\
\text { (Tonne ha-1) }\end{array}$ \\
\hline 1 & $2007-08$ & 2 & 3.11 & 0.78 & 3.90 & 1.94 & 7.15 \\
\hline 2 & $2002-03$ & 4 & 3.55 & 0.88 & 4.43 & 2.21 & 8.12 \\
\hline 3 & 2004-05 & 5 & 21.50 & 5.38 & 26.88 & 13.43 & 49.28 \\
\hline 4 & 2003-04 & 6 & 34.85 & 8.71 & 43.58 & 21.79 & 79.88 \\
\hline 5 & 2001-02 & 8 & 59.11 & 14.77 & 73.88 & 36.94 & 135.44 \\
\hline 6 & $2000-01$ & 9 & 85.18 & 21.30 & 106.47 & 53.24 & 195.20 \\
\hline 7 & $1999-00$ & 10 & 108.65 & 27.16 & 135.81 & 67.90 & 248.99 \\
\hline 8 & $1996-97$ & 13 & 197.86 & 49.47 & 247.32 & 123.67 & 453.43 \\
\hline 9 & $1995-96$ & 14 & 248.48 & 62.13 & 310.61 & 155.30 & 569.44 \\
\hline 10 & $1994-95$ & 15 & 347.56 & 86.89 & 434.43 & 217.22 & 796.48 \\
\hline 11 & $1991-92$ & 18 & 749.14 & 187.29 & 936.42 & 468.20 & 1716.77 \\
\hline
\end{tabular}




\section{Table 3 : Volume, total biomass, carbon stock and $\mathrm{CO}_{2}$ tables of Pongamia pinnata in relation to $\mathrm{DBH}$ and total height of standing trees}

$(\mathrm{DBH}=$ Diameter at breast height in $\mathrm{cm}, \mathrm{VOB}=$ Volume over bark in $\mathrm{m} 3, \mathrm{~TB}=$ Total biomass in tonnes tree ${ }^{-1}, \mathrm{C}=$ Carbon stock in tonnes tre $e^{-1}, \mathrm{CO}_{2}=$ Carbon dioxide in tonnes tree ${ }^{-1}$ )

\begin{tabular}{|c|c|c|c|c|c|c|c|c|c|c|c|c|c|c|}
\hline \multirow{2}{*}{$\begin{array}{l}\text { DBH } \\
(\mathrm{cm})\end{array}$} & \multirow{2}{*}{$\begin{array}{c}\text { Pro- } \\
\text { duction } \\
\text { para- } \\
\text { meters }\end{array}$} & \multicolumn{13}{|c|}{ Total Height (m) } \\
\hline & & 2 & 4 & 6 & 8 & 10 & 12 & 14 & 16 & 18 & 20 & 22 & 24 & 25 \\
\hline \multirow{4}{*}{4} & VOB & 0.0020 & 0.0030 & 0.0040 & 0.0040 & 0.0050 & 0.0060 & 0.0070 & 0.0080 & 0.0090 & 0.0090 & 0.0100 & 0.0110 & 0.0110 \\
\hline & TB & 0.0021 & 0.0031 & 0.0041 & 0.0041 & 0.0051 & 0.0062 & 0.0072 & 0.0082 & 0.0092 & 0.0092 & 0.0103 & 0.0113 & 0.0113 \\
\hline & $\mathrm{C}$ & 0.0010 & 0.0015 & 0.0021 & 0.0021 & 0.0026 & 0.0031 & 0.0036 & 0.0041 & 0.0046 & 0.0046 & 0.0051 & 0.0056 & 0.0056 \\
\hline & $\mathrm{CO}_{2}$ & 0.0038 & 0.0056 & 0.0075 & 0.0075 & 0.0094 & 0.0113 & 0.0132 & 0.0150 & 0.0169 & 0.0169 & 0.0188 & 0.0207 & 0.0207 \\
\hline \multirow{4}{*}{6} & VOB & 0.0070 & 0.0090 & 0.0110 & 0.0130 & 0.0140 & 0.0160 & 0.0180 & 0.0200 & 0.0220 & 0.0240 & 0.0260 & 0.0280 & 0.0280 \\
\hline & TB & 0.0072 & 0.0092 & 0.0113 & 0.0133 & 0.0144 & 0.0164 & 0.0185 & 0.0205 & 0.0226 & 0.0246 & 0.0267 & 0.0287 & 0.0287 \\
\hline & C & 0.0036 & 0.0046 & 0.0056 & 0.0067 & 0.0072 & 0.0082 & 0.0092 & 0.0103 & 0.0113 & 0.0123 & 0.0133 & 0.0144 & 0.0144 \\
\hline & $\mathrm{CO}_{2}$ & 0.0132 & 0.0169 & 0.0207 & 0.0245 & 0.0263 & 0.0301 & 0.0339 & 0.0376 & 0.0414 & 0.0451 & 0.0489 & 0.0527 & 0.0527 \\
\hline \multirow{4}{*}{8} & VOB & 0.0120 & 0.0160 & 0.0190 & 0.0220 & 0.0260 & 0.0290 & 0.0320 & 0.0360 & 0.0390 & 0.0420 & 0.0450 & 0.0490 & 0.0500 \\
\hline & TB & 0.0123 & 0.0164 & 0.0195 & 0.0226 & 0.0267 & 0.0298 & 0.0328 & 0.0369 & 0.0400 & 0.0431 & 0.0462 & 0.0503 & 0.0513 \\
\hline & C & 0.0062 & 0.0082 & 0.0097 & 0.0113 & 0.0133 & 0.0149 & 0.01 & 0.0185 & 0.0200 & 0.0215 & 0.0231 & 0.0251 & 0.0257 \\
\hline & $\mathrm{CO}_{2}$ & 0.0226 & 0.0301 & 0.0357 & 0.0414 & 0.0489 & 0.0545 & 0.0602 & 0.0677 & 0.0734 & 0.0790 & 0.0846 & 0.0922 & 0.0941 \\
\hline \multirow{4}{*}{10} & VOB & 0.0180 & 0.0230 & 0.0290 & 0.0340 & 0.0390 & 0.0440 & 0.0490 & 0.0540 & 0.0590 & 0.0640 & 0.0690 & 0.0740 & 0.0770 \\
\hline & TB & 0.0185 & 0.0236 & 0.0298 & 0.0349 & 0.0400 & 0.0451 & 0.0503 & 0.0554 & 0.0605 & 0.0657 & 0.0708 & 0.0759 & 0.0790 \\
\hline & C & 0.0092 & 0.0118 & 0.0149 & 0.0174 & 0.0200 & 0.0226 & 0.0251 & 0.0277 & 0.0303 & 0.0328 & 0.0354 & 0.0380 & 0.0395 \\
\hline & $\mathrm{CO}_{2}$ & 0.0339 & 0.0433 & 0.0545 & 0.0640 & 0.0734 & 0.0828 & 0.0922 & 0.1016 & 0.1110 & 0.1204 & 0.1298 & 0.1392 & 0.1448 \\
\hline \multirow{4}{*}{12} & VOB & 0.0250 & 0.0320 & 0.0400 & 0.0470 & 0.0540 & 0.0610 & 0.0690 & 0.0760 & 0.0830 & 0.0900 & 0.0970 & 0.1040 & 0.1070 \\
\hline & TB & 0.0257 & 0.0328 & 0.0410 & 0.0482 & 0.0554 & 0.0626 & 0.0708 & 0.0780 & 0.0852 & 0.0923 & 0.0995 & 0.1067 & 0.1098 \\
\hline & C & 0.0128 & 0.0164 & 0.0205 & 0.0241 & 0.0277 & 0.0313 & 0.0354 & 0.0390 & 0.0426 & 0.0462 & 0.0498 & 0.0534 & 0.0549 \\
\hline & $\mathrm{CO}_{2}$ & 0.0470 & 0.0602 & 0.0752 & 0.0884 & 0.1016 & 0.1147 & 0.1 & 0.1430 & 0.1561 & 0.1693 & 0.1825 & 0.1956 & 0.2013 \\
\hline \multirow{4}{*}{14} & VOB & 0.0310 & 0.0410 & 0.0510 & 0.0610 & $\mid 0.0710$ & 0.0810 & 0.0900 & 0.1000 & 0.1090 & 0.1180 & 0.1280 & 0.1370 & 0.1410 \\
\hline & TB & 0.0318 & 0.0421 & 0.0523 & 0.0626 & 0.0728 & 0.0 & 0.0 & 0.1026 & 0.1 & 0.1211 & 313 & 0. & 0.1447 \\
\hline & C & 0.0159 & 0.0210 & 0.0262 & 0.0313 & 0.0 & 0.0 & 0. & 0. & 0.0 & 0.0605 & 0.0657 & 0.0703 & 0.0723 \\
\hline & $\mathrm{CO}_{2}$ & 0.0583 & 0.0771 & 0.0959 & 0.1 & 0.1 & 0.1 & 0. & 0. & 0.2050 & 0.2220 & 0.2 & 0.2577 & 0.2652 \\
\hline \multirow{4}{*}{16} & VOB & 0.0380 & 0.0520 & 0.0650 & 0.0770 & 0.0900 & 0.1020 & 0.1 & 0.1270 & 0.1380 & 0.1500 & 0.1610 & 0.1720 & 0.1780 \\
\hline & TB & 0.0390 & 0.0534 & 0.0667 & 0.0790 & 0.0923 & 0.1047 & 0.1 & 0.1303 & 0.1416 & 0.1539 & 0.1652 & 0.1765 & 0.1826 \\
\hline & C & 0.0195 & 0.0267 & 0.0333 & 0.0395 & 0.0462 & 0.0523 & 0.0 & 0.0652 & 0.0708 & 0.0770 & 0.0826 & 0.0882 & 0.0913 \\
\hline & $\mathrm{CO}_{2}$ & 0.0715 & 0.0978 & 0.1223 & 0.1448 & 0.1693 & 0.1919 & 0.2163 & 0.2389 & 0.2596 & 0.2822 & 0.3028 & 0.3235 & 0.3348 \\
\hline \multirow{4}{*}{18} & VOB & 0.0460 & 0.0630 & 0.0790 & 0.0950 & 0.1100 & 0.1260 & 0.1410 & 0.1550 & 0.1700 & 0.1840 & 0.1970 & 0.2110 & 0.2170 \\
\hline & TB & 0.0472 & 0.0646 & 0.0811 & 0.0975 & 0.1129 & 0.1293 & 0.1447 & 0.1590 & 0.1744 & 0.1888 & 0.2021 & 0.2165 & 0.2226 \\
\hline & C & 0.0236 & 0.0323 & 0.0405 & 0.0487 & 0.0564 & 0.0646 & 0.0723 & 0.0795 & 0.0872 & 0.0944 & 0.1011 & 0.1082 & 0.1113 \\
\hline & $\mathrm{CO}_{2}$ & 0.0865 & 0.1185 & 0.1486 & 0.1787 & 0.2069 & 0.2370 & 0.2652 & 0.2916 & 0.3198 & 0.3461 & 0.3706 & 0.3969 & 0.4082 \\
\hline \multirow{4}{*}{20} & VOB & 0.0540 & 0.0740 & 0.0940 & 0.1130 & 0.1320 & 0.1510 & 0.1690 & 0.1860 & 0.2030 & 0.2190 & 0.2350 & 0.2510 & 0.2580 \\
\hline & TB & 0.0554 & 0.0759 & 0.0964 & 0.1159 & 0.1354 & 0.1549 & 0.1734 & 0.1908 & 0.2083 & 0.2247 & 0.2411 & 0.2575 & 0.2647 \\
\hline & C & 0.0277 & 0.0380 & 0.0482 & 0.0580 & 0.0677 & 0.0775 & 0.0867 & 0.0954 & 0.1041 & 0.1123 & 0.1206 & 0.1288 & 0.1324 \\
\hline & $\mathrm{CO}_{2}$ & & & & 0.2126 & & & & & & & 0.4420 & & 0.4853 \\
\hline \multirow{4}{*}{22} & VOB & 0.0620 & 0.0870 & 0.1100 & 0.1330 & 0.1560 & 0.1770 & 0.1 & 0.2180 & 0.2380 & 0.2560 & 0.2740 & 0.2910 & 0.3000 \\
\hline & TB & 0.0636 & 0.0893 & 0.1129 & 0.1365 & 0.1601 & 0.1816 & 0.2031 & 0.2237 & 0.2442 & 0.2627 & 0.2811 & 0.2986 & 0.3078 \\
\hline & C & 0.0318 & 0.0446 & 0.0564 & 0.0682 & 0.0800 & 0.0908 & 0.1016 & 0.1118 & 0.1221 & 0.1313 & 0.1406 & 0.1493 & 0.1539 \\
\hline & $\mathrm{CO}_{2}$ & 0.1166 & 0.1636 & 0.2069 & 0.2502 & 0.2934 & 0.3329 & 0.3724 & 0.4101 & 0.4477 & 0.4815 & 0.5154 & 0.5474 & 0.5643 \\
\hline \multirow{4}{*}{24} & VOB & 0.0710 & 0.1000 & 0.1280 & 0.1540 & 0.1800 & 0.2050 & 0.2290 & 0.2510 & 0.2730 & 0.2940 & 0.3130 & 0.3320 & 0.3410 \\
\hline & TB & 0.0728 & 0.1026 & 0.1313 & 0.1580 & 0.1847 & 0.2103 & 0.2350 & 0.2575 & 0.2801 & 0.3016 & 0.3211 & 0.3406 & 0.3499 \\
\hline & C & 0.0364 & 0.0513 & 0.0657 & 0.0790 & 0.0923 & 0.1052 & 0.1175 & 0.1288 & 0.1400 & 0.1508 & 0.1606 & 0.1703 & 0.1749 \\
\hline & $\mathrm{CO}_{2}$ & 0.1336 & 0.1881 & 0.2408 & 0.2897 & 0.3386 & 0.3856 & 0.4307 & 0.472 & 0.5135 & 0.5530 & 0.5888 & 0.6245 & 0.6414 \\
\hline
\end{tabular}




\begin{tabular}{|c|c|c|c|c|c|c|c|c|c|c|c|c|c|c|}
\hline \multirow{2}{*}{$\begin{array}{l}\text { DBH } \\
(\mathrm{cm})\end{array}$} & \multirow{2}{*}{$\begin{array}{c}\text { Pro- } \\
\text { duction } \\
\text { para- } \\
\text { meters }\end{array}$} & \multicolumn{13}{|c|}{ Total Height (m) } \\
\hline & & 2 & 4 & 6 & 8 & 10 & 12 & 14 & 16 & 18 & 20 & 22 & 24 & 25 \\
\hline \multirow{4}{*}{26} & VOB & 0.0800 & 0.1140 & 0.1460 & 0.1760 & 0.2060 & 0.2340 & 0.2600 & 0.2850 & 0.3090 & 0.3310 & 0.3520 & 0.3710 & 0.3800 \\
\hline & TB & 0.0821 & 0.1170 & 0.1498 & 0.1806 & 0.2114 & 0.2401 & 0.2668 & 0.2924 & 0.3170 & 0.3396 & 0.3612 & 0.3806 & 0.3899 \\
\hline & $\mathrm{C}$ & 0.0410 & 0.0585 & 0.0749 & 0.0903 & 0.1057 & 0.1200 & 0.1334 & 0.1462 & 0.1585 & 0.1698 & 0.1806 & 0.1903 & 0.1949 \\
\hline & $\mathrm{CO}_{2}$ & 0.1505 & 0.2144 & 0.2746 & 0.3311 & 0.3875 & 0.4402 & 0.4891 & 0.5361 & 0.5812 & 0.6226 & 0.6621 & 0.6979 & 0.7148 \\
\hline \multirow{4}{*}{28} & VOB & 0.0890 & 0.1280 & 0.1650 & 0.1990 & 0.2320 & 0.2630 & 0.2920 & 0.3190 & 0.3440 & 0.3680 & 0.3890 & 0.4090 & 0.4180 \\
\hline & TB & 0.0913 & 0.1313 & 0.1693 & 0.2042 & 0.2380 & 0.2698 & 0.2996 & 0.3273 & 0.3529 & 0.3776 & 0.3991 & 0.4196 & 0.4289 \\
\hline & $\mathrm{C}$ & 0.0457 & 0.0657 & 0.0846 & 0.1021 & 0.1190 & 0.1349 & 0.1498 & 0.1636 & 0.1765 & 0.1888 & 0.1996 & 0.2098 & 0.2144 \\
\hline & $\mathrm{CO}_{2}$ & 0.1674 & 0.2408 & 0.3104 & 0.3743 & 0.4364 & 0.4947 & 0.5493 & 0.6000 & 0.6471 & 0.6922 & 0.7317 & 0.7693 & 0.7863 \\
\hline \multirow{4}{*}{30} & VOB & 0.0990 & 0.1430 & 0.1840 & 0.2230 & 0.2590 & 0.2930 & 0.3240 & 0.3530 & 0.3790 & 0.4030 & 0.4240 & 0.4430 & 0.4510 \\
\hline & TB & 0.1016 & 0.1467 & 0.1888 & 0.2288 & 0.2657 & 0.3006 & 0.3324 & 0.3622 & 0.3889 & 0.4135 & 0.4350 & 0.4545 & 0.4627 \\
\hline & C & 0.0508 & 0.0734 & 0.0944 & 0.1144 & 0.1329 & 0.1503 & 0.1662 & 0.1811 & 0.1944 & 0.2067 & 0.2175 & 0.2273 & 0.2314 \\
\hline & $\mathrm{CO}_{2}$ & 0.1862 & 0.2690 & 0.3461 & 0.4195 & 0.4872 & 0.5511 & 0.6094 & 0.6640 & 0.7129 & 0.7580 & 0.7975 & 0.8333 & 0.8483 \\
\hline \multirow{4}{*}{32} & VOB & 0.1090 & 0.1590 & 0.2040 & 0.2470 & 0.2870 & 0.3230 & 0.3560 & 0.3860 & 0.4120 & 0.4350 & 0.4550 & 0.4720 & 0.4790 \\
\hline & TB & 0.1118 & 0.1631 & 0.2093 & 0.2534 & 0.2945 & 0.3314 & 0.3653 & 0.3960 & 0.4227 & 0.4463 & 0.4668 & 0.4843 & 0.4915 \\
\hline & C & 0.0559 & 0.0816 & 0.1047 & 0.1267 & 0.1472 & 0.1657 & $0.1826 \mid$ & 0.1980 & 0.2114 & 0.2232 & 0.2334 & 0.2421 & 0.2457 \\
\hline & $\mathrm{CO}_{2}$ & 0.2050 & 0.2991 & 0.3837 & 0.4646 & 0.5398 & 0.6076 & 0.6696 & 0.7261 & 0.7750 & 0.8182 & 0.8559 & 0.8878 & 0.9010 \\
\hline \multirow{4}{*}{34} & VOB & 0.1200 & 0.1750 & 0.2250 & 0.2720 & 0.3140 & 0.3530 & 0.3 & 0.4170 & 0.4430 & 0.4640 & 0.4820 & 0.4960 & 0.5010 \\
\hline & TB & 0.1231 & 0.1796 & 0.2309 & 0.27 & 0.3222 & 0.3622 & 0.3 & 0.4 & 0.4 & 0.4 & 0.4945 & 89 & 0.5140 \\
\hline & C & 0.0616 & 0.0898 & 0.1154 & 0.1 & 0.1 & 0.1811 & & 0.2139 & 0.2273 & 0.2380 & 0.2473 & 0.2544 & 0.2570 \\
\hline & $\mathrm{CO}_{2}$ & 0.2257 & 0.3292 & 0.4232 & 0.5116 & 0.5906 & 0.6640 & 0.7279 & 0.7844 & 0.8333 & 0.8728 & 0.9066 & 0.9330 & 0.9424 \\
\hline \multirow{4}{*}{36} & VOB & 0.1310 & 0.1910 & 0.2470 & 0.2970 & 0.3420 & 0.3820 & 0.4 & 0.4460 & 0.4700 & 0.4890 & 0.5030 & 0.5120 & 0.5140 \\
\hline & TB & 0.1344 & 0.1960 & 0.2534 & 0.3 & 0.3509 & 0.3919 & 0.4 & 0.4576 & 0.4822 & 0.5017 & 0.5161 & 0.5253 & 0.5274 \\
\hline & $\mathrm{C}$ & 0.0672 & 0.0980 & 0.1267 & 0.1524 & 0.1754 & 0.1960 & 0.2 & 0.2288 & 0.2411 & 0.2509 & 0.2580 & 0.2627 & 0.2637 \\
\hline & $\mathrm{CO}_{2}$ & 0.2464 & 0.3593 & 0.4646 & 0.5587 & 0.6433 & 0.7185 & 0.7 & 0.8389 & 0.8841 & 0.9198 & 0.9461 & 0.9631 & 0.9668 \\
\hline \multirow{4}{*}{38} & VOB & 0.1420 & 0.2080 & 0.2690 & 0.3220 & 0.3690 & 0.4100 & 0.4440 & 0.4720 & 0.4940 & 0.5090 & 0.5170 & 0.5190 & 0.5180 \\
\hline & TB & 0.1457 & 0.2134 & 0.2760 & 0.3304 & 0.3786 & 0.4207 & 0.45 & 0.4843 & 0.5068 & 0.5222 & 0.5304 & 0.5325 & 0.5315 \\
\hline & $\mathrm{C}$ & 0.0728 & 0.1067 & 0.1380 & 0.1652 & 0.1893 & 0.2103 & 0.2278 & 0.2421 & 0.2534 & 0.2611 & 0.2652 & 0.2662 & 0.2657 \\
\hline & $\mathrm{CO}_{2}$ & 0.2671 & 0.3912 & 0.5060 & 0.6057 & 0.6941 & 0.7712 & 0.8352 & 0.8878 & 0.9292 & 0.9574 & 0.9725 & 0.9762 & 0.9744 \\
\hline \multirow{4}{*}{40} & VOB & 0.1530 & 0.2260 & 0.2910 & 0.3470 & 0.3960 & 0.4370 & 0.4700 & 0.4950 & 0.5120 & 0.5220 & 0.5230 & 0.5160 & 0.5100 \\
\hline & TB & 0.1570 & 0.2319 & 0.2986 & 0.3560 & 0.4063 & 0.4484 & 0.4822 & 0.5079 & 0.5253 & 0.5356 & 0.5366 & 0.5294 & 0.5233 \\
\hline & C & 0.0785 & 0.1159 & 0.1493 & 0.1780 & 0.2031 & 0.2242 & 0.2411 & 0.2539 & 0.2627 & 0.2678 & 0.2683 & 0.2647 & 0.2616 \\
\hline & $\mathrm{CO}_{2}$ & 0.2878 & 0.4251 & 0.5474 & 0.6527 & 0.7449 & 0.8220 & 0.8 & 0.9311 & 0.9 & 0.9819 & 0.9838 & 0.9706 & 0.9593 \\
\hline \multirow{4}{*}{42} & VOB & 0.1650 & 0.2440 & 0.3130 & 0.3720 & 0.4220 & 0.4620 & 0.4 & 0.5140 & 0.5250 & 0.5270 & 0.5190 & 0.5010 & 0.4890 \\
\hline & TB & 0.1693 & 0.2503 & 0.3211 & 0.38 & 0.4 & 0.4740 & 0.5 & 0.5274 & 0.5387 & 0.5407 & 0.5325 & 0.5140 & 0.5017 \\
\hline & C & 0.0846 & 0.1252 & 0.1606 & 0.19 & 0.2165 & 0.2370 & 0.2 & 0.2637 & 0.2693 & 0.2704 & 0.2662 & 0.2570 & 0.2509 \\
\hline & $\mathrm{CO}_{2}$ & 0.3104 & 0.4590 & 0.5888 & 0.6997 & 0.7 & 0.8690 & 0.9 & 0.9668 & 0.9875 & 0.9913 & 0.9762 & 0.9424 & 0.9198 \\
\hline \multirow{4}{*}{44} & VOB & 0.1770 & 0.2620 & 0.3350 & 0.3 & 0.4470 & 0.4850 & 0.5 & 0.5270 & 0.5310 & 0.5230 & 0.5040 & 0.4730 & 0.4530 \\
\hline & TB & 0.1816 & 0.2688 & 0.3437 & 0.4073 & 0.4586 & 0.4976 & 0.5 & 0.5407 & 0.5448 & 0.5366 & 0.5171 & 0.4853 & 0.4648 \\
\hline & C & 0.0908 & 0.1344 & 0.1719 & 0.2037 & 0.2293 & 0.2488 & 0.2627 & 0.2704 & 0.2724 & 0.2683 & 0.2586 & 0.2426 & 0.2324 \\
\hline & $\mathrm{CO}_{2}$ & 0.3329 & 0.4928 & 0.6301 & 0.7468 & 0.8408 & 0.9123 & 0.9631 & 0.9913 & 0.9988 & 0.9838 & 0.9480 & 0.8897 & 0.8521 \\
\hline \multirow{4}{*}{46} & VOB & 0.1900 & 0.2810 & 0.3580 & 0.4210 & 0.4700 & 0.5060 & 0.5270 & 0.5350 & 0.5290 & 0.5100 & 0.4760 & 0.4280 & 0.3990 \\
\hline & TB & 0.1949 & 0.2883 & 0.3673 & 0.4319 & 0.4822 & 0.5192 & 0.5407 & 0.5489 & 0.5428 & 0.5233 & 0.4884 & 0.4391 & 0.4094 \\
\hline & C & 0.0975 & 0.1442 & 0.1837 & 0.2160 & 0.2411 & 0.2596 & 0.2704 & 0.2745 & 0.2714 & 0.2616 & 0.2442 & 0.2196 & 0.2047 \\
\hline & $\mathrm{CO}_{2}$ & 0.3574 & 0.5286 & 0.6734 & 0.79 & 0.8841 & 0.9518 & 0.9913 & 1.0063 & 0.9950 & 0.9593 & 0.8954 & 0.8051 & 0.7505 \\
\hline \multirow{4}{*}{48} & VOB & 0.2020 & 0.2990 & 0.3800 & 0.4440 & 0.4920 & 0.5230 & 0.5380 & 0.5370 & 0.5190 & 0.4840 & 0.4340 & 0.3670 & 0.3270 \\
\hline & TB & 0.2073 & 0.3068 & 0.3899 & 0.4555 & 0.5048 & 0.5366 & 0.5520 & 0.5510 & 0.5325 & 0.4966 & 0.4453 & 0.3765 & 0.3355 \\
\hline & C & 0.1036 & 0.1534 & 0.1949 & 0.2278 & 0.2524 & 0.2683 & 0.2760 & 0.2755 & 0.2662 & 0.2483 & 0.2226 & 0.1883 & 0.1678 \\
\hline & $\mathrm{CO}_{2}$ & 0.3800 & 0.5624 & 0.7148 & 0.8352 & 0.9255 & 0.9838 & 1.0120 & 1.0101 & 0.9762 & 0.9104 & 0.8164 & 0.6903 & 0.6151 \\
\hline & VOB & 0.2150 & 0.3180 & 0.4020 & 0.4660 & 0.5110 & 0.5370 & 0.5430 & 0.5300 & 0.4980 & 0.4460 & 0.3750 & 0.2850 & 0.2330 \\
\hline . & TB & 0.2206 & 0.3263 & 0.4125 & 0.4781 & 0.5243 & 0.5510 & 0.5571 & 0.5438 & 0.5109 & 0.4576 & 0.3848 & 0.2924 & 0.2391 \\
\hline 5 & $\mathrm{C}$ & 0.1103 & 0.1631 & 0.2062 & 0.2391 & 0.2621 & 0.2755 & 0.2786 & 0.2719 & 0.2555 & 50.2288 & 0.1924 & 0.1462 & 0.1195 \\
\hline & $\mathrm{CO}_{2}$ & 0.4044 & 0.5982 & 0.7562 & 0.8765 & 0.9612 & 1.0101 & 1.0214 & 0.9969 & 0.9367 & 70.8389 & 0.7054 & 0.5361 & 0.4383 \\
\hline
\end{tabular}




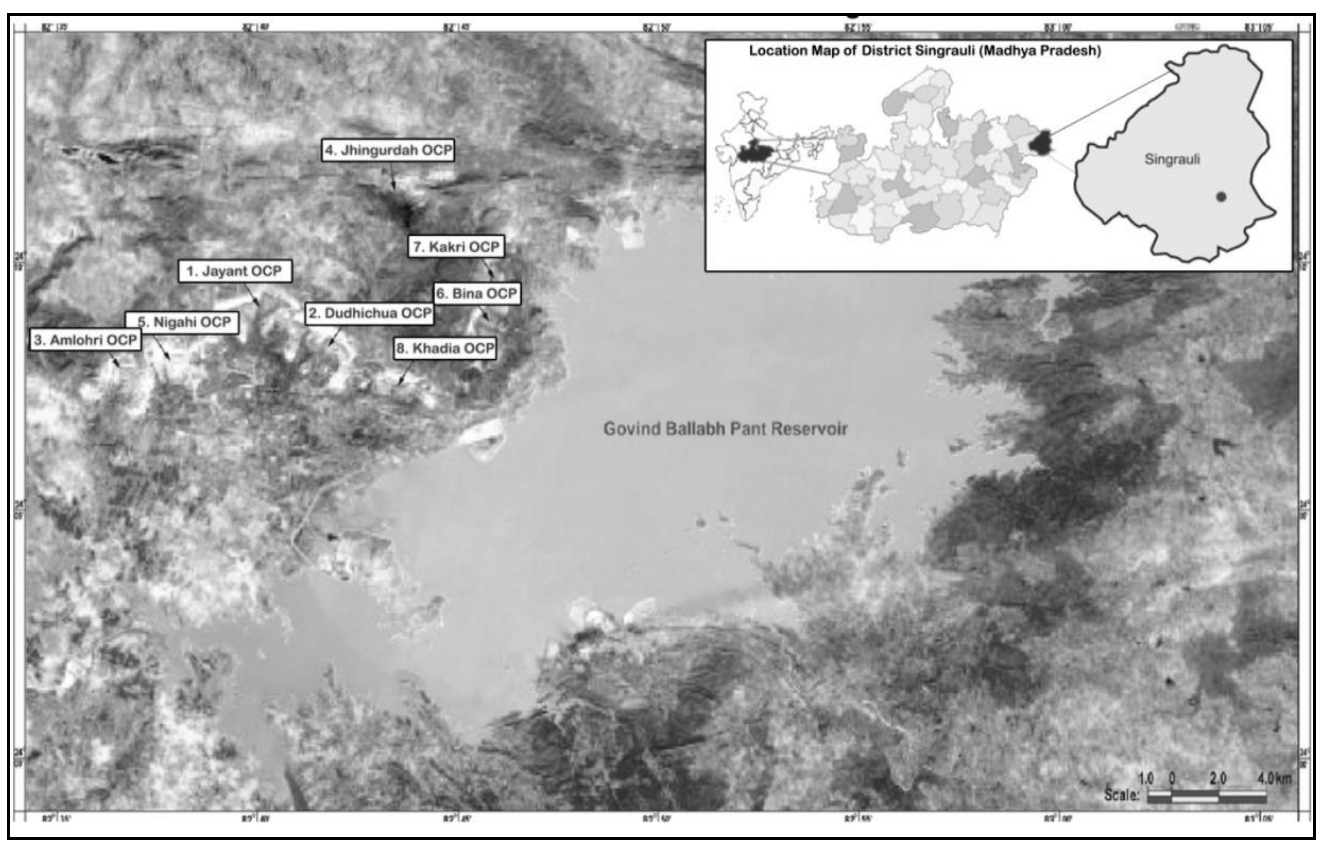

Figure 1. Satellite imagery of the study area showing different opencast project (OCP) site and Govind Ballabh Pant Reservoir at Singrauli, India

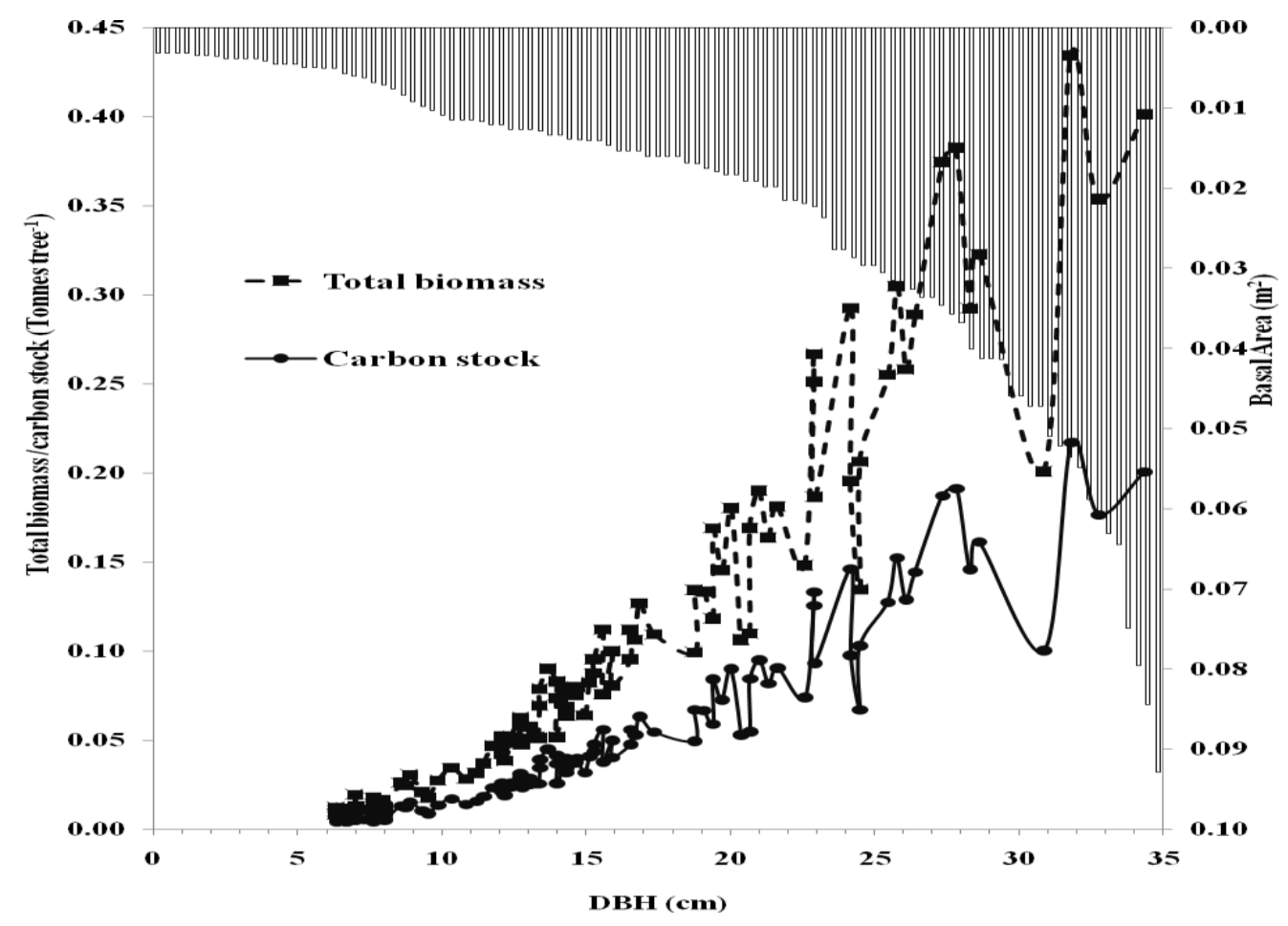

Figure 2. Correlation among different growth parameters pertaining to DBH, basal area, total biomass and carbon stock in Pongamia pinnata 


\section{Discussion}

Perusal of data on biomass accumulation (above ground, below ground and total biomass) showed increasing trend with $\mathrm{dbh}$ and height classes. The volume and subsequently, biomass was reported to be related with diameter (dbh) and height (H) (FSI 1996). For determining the best fit equation of volume, independent variables (diameter and height) such as $\mathrm{D}, \mathrm{D}^{2} \mathrm{H}$ and $\left(\mathrm{D}^{2} \mathrm{H}\right)^{2}$ were used for Pongamia pinnata. The range of determination coefficient was found to be $94 \%$. This could be explained by the fact that volume $(\mathrm{V})$ and above ground components of trees were dependent upon dbh and height (Bohre et al., 2012).

In terms of vertical and horizontal growth, Pongamia pinnata proved as an efficient species with the corresponding heights and dbh of $12.15 \mathrm{~m}$ and $27.8 \mathrm{~cm}$, respectively in 18 years old plantations. During initial growth period, the performance of Pongamia pinnata was better in terms of both vertical and horizontal growth (Bohre et. al., 2013). This was primarily due to their nitrogen fixing nature. Most of the leguminous species form nodules in the root with the symbiotic association of Rhizobium species and fixes atmospheric nitrogen. About 57\% of the legume genera comprising 3395 species were examined with nodulation. Of the examined species, about 23\% species belonged to Caesalpiniaceae, $97 \%$ to Papilionaceae and 90\% to Mimosaceae (Srivastava et al., 1999). It might be due to high energy conversion efficiency of these species. The energy conversion efficiency may be defined as the ratio of amount of photosynthetically active radiation (PAR) that falls on the plant canopy to the amount of energy fixed by the plant. The efficiency of utilization of solar energy at cellular level was quite high being 30\% (Kok, 1973; Kok et al., 1976). In the present study, the biomass accumulation in Pongamia pinnata takes over the lead successive development. Though, the nature of biomass accumulation may vary in the same species under different localities under such factors as accumulation of nutrient in the soil, management practices and availability of light and other environmental factors. The results were in agreement with the findings of Chaturvedi and Behl (1996), Goel and Behl (1999a,b, 2004, 2005), Singh and Goel (2009) who estimated the production potential of exotic and indigenous tree species of degraded soil sites under sodicity stress conditions. The better performance of this species in plantation forests might be primarily due to well drained and highly porous texture of soil media as found in mined overburdens.

The net biomass production of Pongamia pinnata showed increasing trend with the age (from 2 to 18 years) of plantations. The findings were comparable with the results of Datta and Agarwal (2003), Karmacharya and Singh (1992), Buvaneswaran et al., (2006); Nandeswar et al., (1996), Pozgaj et al., (1996), Leith et al., (1986) Bohre et al., (2012, 2013), Chaubey et al., (2012).

\section{Conclusions}

Looking to the serious concern of carbon management, the role of Pongamia pinnata to estimate the biomass accumulation and its contribution for sequestration of carbon in mined out areas, the following conclusions were derived:

1. Actual volume resembled closely with predicted volumes and showed significantly increasing trend with the increase in the height and diameter of trees.

2. The best fit allometric equations developed for volume estimation and subsequently biomass accumulation and carbon sequestration adopting non harvest technique for Pongamia pinnata being VOB $=-0.007+0.002 \mathrm{D}+2.638 \times 10^{-5} \mathrm{D}^{2} \mathrm{H}-3.863 \times 10^{-10}$ $\left(\mathrm{D}^{2} \mathrm{H}\right)^{2} ; \mathrm{R}^{2}=0.940 ; \mathrm{SE}=0.0235515$. 


\section{References}

[1] P. Bohre, O. P. Chaubey and P. K. Singhal, "Biomass accumulation and carbon sequestration in Dalbergia sissoo Roxb", International Journal of Bio-Science and Bio-Technology, vol. 4, no. 3, (2012a), pp. 29-44.

[2] P. Bohre, O. P. Chaubey and P. K. Singhal, "Bio-restoration and its impact on species diversity and biomass accumulation of ground flora community of degraded ecosystem of coalmines", International Journal of BioScience and Bio-Technology, vol. 4, no. 4, (2012b), pp. 63-79.

[3] P. Bohre, O. P. Chaubey and P. K. Singhal, "Biomass production and carbon sequestration by Cassia siamea Lamk in degraded ecosystem", In: O.P. Chaubey and Ram Prakash (ed.), Sustainable bio-diversity conservation in the landscape, Aavishkar publishers, distributors, Jaipur, India, (2013), pp. 15-34.

[4] S. Brown and A. E. Lugo, "Aboveground biomass estimates for tropical moist forests of the Brazilian Amazon", Jaterciercia, vol. 17, no. 1, (1992), pp. 8-18.

[5] C. Buvaneswaran, M. George, D. Perez and M. Kanninen, "Biomass of teak plantations in Tamil Nadu, India and Costa Rica compared”, Journal of Tropical Forest Science, vol. 18, no. 3, (2006), pp. 195-197.

[6] O. P. Chaubey, P. Bohre and P. K. Singhal, "Impact of bio-reclamation of coal mine spoil on nutritional and microbial characteristics - a case study”, International Journal of Bio-Science and Bio-Technology, vol. 4, no. 3, (2012), pp. 69-79.

[7] A. N. Chaturvedi and H. M. Behl, "Biomass production trials on sodic sites", Indian Forester, vol. 122, no. 6, (1996), pp. 439-455.

[8] R. K. Dutta and M. Agarwal, "Effect of tree plantations on the soil characteristics and microbial activity of coal mine spoil land", Tropical Ecology, vol. 43, no. 2, (2002), pp. 315-324.

[9] P. J. Edwards and P. J. Grubbs, "Studies of mineral cycling in a Montane Rain Forest in New Guinea, IDistribution of organic matter in the vegetation and soil", J. Ecol., vol. 65, (1977), pp. 1943-1969.

[10] N. J. Enright, "Litter production and nutrient partitioning in rain forest near Bulolo", Papa New Guinea, Malay For., vol. 42, (1979), pp. 202-209.

[11] Forest Survey of India, Volume equations for forests of India, Nepal and Bhutan. Published by Director, Forest Survey of India Dehra Dun- 248195 and Printed at Saraswati Press Dehra Dun - 248001, (1996), pp. 249.

[12] GIM, moef.nic.in/downloads/public-information/GIM-Report-PMCCC.pdf, (2012) July 12.

[13] V. L. Goel and H. M. Behl, "Evaluation of Sesbania formosa for rehabilitation of degraded sodic soils", Forest, Farm and Community Tree Research Report, vol. 4, (1999a), pp. 112-116.

[14] V. L. Goel and H. M. Behl, "Screening of Prosopis germplasm for afforestation of degraded soil sites: Performance, leaf nutrient status and influence on sil properties", J. Sustainable Forestry, vol. 8, no. 2, (1999b), pp. 1-13.

[15] V. L. Goel and H. M. Behl, "Productivity assessment of three leguminous species under high density plantations on degraded soil sites", Bimass Bioenergy, vol. 27, no. 5, (2004), pp. 403-406.

[16] V. L. Goel and H. M. Behl, "Growth and productivity assessment of Casuarina glauca Sieb. Ex. Spreng on sodic soils sites", Bioresource Technology, vol. 96, (2005), pp. 1399-1404.

[17] T. C. Hannan, "Rehabilitation of large-scale opencast coalmines", Jour. of Soil Conser. Service, NSW, vol. 35, no. 4, (1979), pp. 184-193.

[18] P. Heilman, "Effect of surface treatment and interplanting of shrub older on growth of Douglas fir on coal soils", Jour. of Envi., vol. 12, no. 1, (1983), pp. 109-113.

[19] K. Hozumi, K. Yoda and T. Kira, "Production ecology of tropical rain forests in southern western Combodia. II- Photosynthetic prodctuin in evergreen seasonal forests", Nature and life in South East Asia, vol. 6, (1969), pp. 57-81.

[20] Intergovernmental Panel on Climate Change (IPCC), Guidelines for national greenhouse gas inventories, In: Simon Eggelston, Leandro Buendia, Kyoko Miwa, Todd Ngara, Kiyoto Tanabe (eds.), The institute for global environmental strategies (IGES) for the IPCC ISBN 4-88788-032-4, (2006).

[21] International Symposium on Feedstock Recycling (ISFR), Proceedings of the $6^{\text {th }}$ international symposium on feedstock recycling of polymeric materials, Toledo (Spain), (2011) October 5-7.

[22] C. F. Jordan, "Productivity of tropical forest and its relation to a world pattern of energy storage", J. Ecol., vol. 59, (1971), pp. 127-142.

[23] S. B. Karmacharya and K. P. Singh, "Biomass and net production of teak plantations in a dry tropical region in India", Forest Ecology and Management, vol. 55, (1992), pp. 233-247.

[24] T. Kira, H. Ogawa, K. Yoda and K. Ogino, "Primary production by a Tropical Rain Forest of Southern Thailand", Bot. Mag., vol. 77, (1964), pp. 425-429.

[25] B. Kok, "Photosynthesis", Proceedings of the workshop on Bio-solar conversion (eds. Gibbs, M., Holaender, A., Kok, B. Krampitz, L.O. and Pietro, A, San) NSF-RANN Report, (1973), pp. 22. 
[26] B. Kok, C. F. Fowler, H. H. Hardt and R. J. Radmer, "Biological solar energy conversion: approaches to overcome yield stability and product limitations", Enzyme technology and renewable resources, (eds. Grainer, J.L.) University of Virginia and NSF-RANN, (1976).

[27] J. H. Leith, J. P. Reynolds and H. H. Rogers, "Estimation of leaf area of Soybeans grown under elevated carbon dioxide levels", Field Crops Res., vol. 13, (1986), pp. 193-203.

[28] Manua Loa Observatory, "Trends in atmospheric carbon dioxide. Recent Mauna Loa $\mathrm{CO}_{2}$ ", http://www.esrl.noaa.gov/gmd/ccgg/trends, (2013).

[29] D. L. Nandeswar, D. Dugaya, T. K. Mishra, A. J. Williams and S. K. Banerjee, "Natural succession of an age series of coal mine spoil in sub-tropical region”, Advances in Plant Science Research India, vol. 3, (1996), pp. $105-124$

[30] J. D. S. Negi, N. K. S. Bora, V. N. Tandon and H. D. Thapliyal, "Organic matter production in an age series of Eucalyptus globulus plantations in Tamil Nadu", Indian Forester, vol. 110, no. 8, (1984), pp. 802-813.

[31] H. T. Odum and R. F. Pigeon, "A study of irradiation and ecology at El Verde, Puerio Rico", A tropical Rain Forest., U.S. Atomic Energy Commission, Oak Ridge, Tennessee, (1970), pp. 1-1678.

[32] H. Ogawa, K. Yoda, T. Kira, K. Ogino, T. Shidei, D. Ratanawongs and C. Apasutaya, "Comparative ecological studies on three main types of forest vegetation in Thailand, I-Structure and floristic composition", Nature \& Life in South East Asia, vol. 4, (1965), pp. 13-48.

[33] A. Pozga, A. Iqbal and L. J. Kucera, "Development structure and properties of wood from trees affected by air pollution", In: M. Yunus and M. Iqbal (ed.), Plant Response to Air Pollution. John Willey \& Sons Ltd., (1996), pp. 395-424.

[34] R. Prasad and G. P. Mishra, "Standing biomass of various plant parts in selected tree species of dry deciduous teak forest in M.P.”, Indian Forester, vol. 110, no. 8, (1984), pp. 765-782.

[35] R. Prasad, A. K. Sah, A. S. Bhandari and O. P. Chaubey, "Dry matter production by Eucalyptus camaldulensis Dehn plantation in Jabalpur", Indian Forester, vol. 110, no. 9, (1984), pp. 868-878.

[36] S. N. Rai, "Above ground biomass in torpical rain forests of Western Ghats, India", Indian Forester, vol. 110, no. 8, (1984), pp. 754-764.

[37] N. H. Ravindranath, N. Srivastava, I. K. Murthy, S. Malaviya, M. Munsi and N. Sharma, "Deforestation and forest degradation in India implications of REDD+", Curr. Sci., vol. 102, no. 8, (2012), pp. 1-9.

[38] G. Reyes, S. Brown, J. L. Chapman and E. Ariel, "Wood densities of Tropical tree species", General technical report So-88, United States Department of Agriculture, Forest Service, Southern Forest Experiment Station, New Orieans, Louisiana, (1992).

[39] S. C. Sharma and V. K. Srivastava, "Biomass production in an age series of Pinus patula plantation in Tamil Nadu”, Indian Forester, vol. 110, no. 9, (1984), pp. 915-930.

[40] B. Singh and V. L. Goel, "Rehabilitation of degraded soil sites through afforestation programmes: A case study”, In: O. P. Chaubey, Vijay Bahadur and P. K. Shukla (ed.), Sustainable Rehabilitation of Degraded Ecosystems. Aavishkar Publishers, Distributors Jaipur, Raj. 302003 India, (2009), pp. 67-76.

[41] K. K. Srivastava, R. Prabhakaran and N. Verma, "Effect of nodulation on biomass of some arid zone tree species”, Indian Forester, vol. 125, no. 12, (1999), pp. 1265-1268.

[42] R. Sud, J. V. Sharma and A. K. Bansal, "International REDD+ architecture and its relevance for India", Proceedings of national level consultation on International REDD plus architecture and its relevance for India held on 14th June, 2012, Organized by: MOEF, GoI and TERI, (2012) June 14, pp. 1-11.

[43] E. V. J. Tanner, "Studies on biomass productivity in a series of montane Rain Forest Jamaica", J. Ecol., vol. 68, (1980), pp. 578-588.

[44] T. C. Whitemore, “Tropical Rain Forests of the East”, Clarendon Press, Oxford, London, (1975), pp. pp. 238.

\section{Authors}

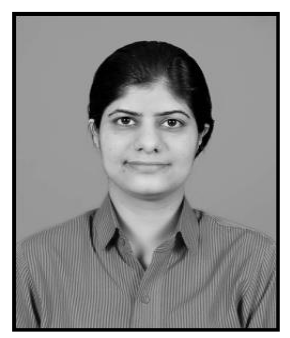

\section{Dr. Prianka Bohre}

Dr. Prianka Bohre has been awarded her Ph.D degree in 2013 on the topic "Biomass accumulation and carbon sequestration by dominant plantation species raised on coal mine overburden at Singrauli" from Rani Durgawati University, Jabalpur (M.P.) India. She did M.Sc in botany (2007-09) and secured first position in III \& IV semester from Govt. Autonomous Holkar Science College, Indore (M.P.) India. She had basic trainings in Recombinant DNA technology \& PCR held in January 2005 at GENEI, Bangalore, India, One module of the Bioscience 


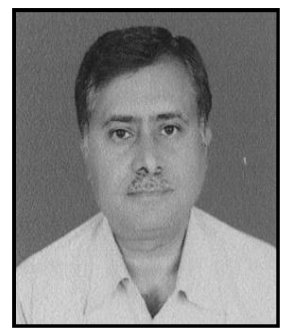

Excellence Graduate Training program on protein and proteomic analysis and molecular \& genomic studies at G-Bioscience, New Delhi, India and graduate training program on biotechnology (September2005) organized at Genetic and Plant Propagation Division of Tropical Forest Research Institute, Jabalpur, India. She has to her credit two awards viz., State Level Award (Rajya Puraskar), 28 March 2001: as a Guide, Kendriya Vidyalaya Sanghatans and Tritiya Sopan Testing Awarded, Kendriya Vidyalaya C.O.D, Jabalpur, 4-7 November 1998: as a guide of Kendriya vidyalaya no-2 GCF, Jabalpur. She has 11 papers published in reputed the International and National scientific journals. She has qualified GRE $\&$ TOEFL by securing- $1270 / 1600$ and $82 / 100$ marks respectively. She want to pursue postdoctoral in United States university to enhance my knowledge in the field of plant science and contribute in the academic society of the United States as a researcher in your university, which offers both - a healthy environment in learning and excellent opportunities for research in the field of Plant Science. She would like to implement the knowledge gained for the benefit of society at large. She is open to further research studies on projects of the department for my academic development.

\section{Dr. O. P. Chaubey}

Dr. O.P. Chaubey is working as Head of Forest Botany Branch in M.P. State Forest Research Institute, Jabalpur (M.P.) India. He was awarded in Ph.D. degree in Forest Ecology in 1986 from Dr. H.S. Gaur University, Sagar, (Madhya Pradesh, India). He has to his credit three books, 13 monograph of various forestry species and more than 75 research papers published in both National and International journals. He has 30 years of research experience in field of forestry. He has completed more than 22 externally funded research projects in the capacity of Principal Investigator. He has organized a number of symposia/ workshops at National and State levels. He has imparted trainings to field foresters, University scholars, NGOs and Rural Communities engaged in conservation and management of biological diversity.

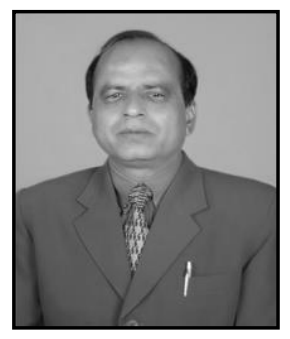

\section{Dr. P. K. Singhal}

Dr. P.K. Singhal is currently working as Professor at Department of Biological Science, Rani Durgavati University, Jabalpur, India. An alumnus of Agra and Saugor Universities, he is actively engaged in research and teaching for the last 30 years in areas of environmental biology and biostatistics, and has published more than 40 research papers and two books. His current research areas are carbon sequestration by natural and artificial plantations, nutrient dynamics in habitats and biotechnological exploitation of microbial enzymes. 